\title{
Crack identification basd on natural frequency of cracked small pipes
}

\author{
Jiuhong Jia*, Xiaoyu Zheng, Zhongqiang Zhang \\ ${ }^{1}$ Key Laboratory of Pressure Systems and Safety, Ministry of Education, \\ East China University of Science and Technology, Shanghai, 200237, China \\ jhiia@ecust.edu.cn, 13701898499@163.com, zzqecust2013@163.com
}

\begin{abstract}
Keywords: Small branch pipe; Natural frequency; Vibration; crack; Nuclear power plant Abstract. The severe vibration of small branch pipes in nuclear power plants can induce fatigue destruction. Sometime fatigue destructions will result in unplanned shutdown, and even threaten the safe and stable operation of the nuclear power plant. In order to predict the remaining life and guarantee the safety of small branch pipes in succeed service, the damage identification basing on vibration frequency is studied in the present research. Cracked small pipes are modeled using the finite element software ABAQUS to analyze their vibration characteristics. The first four natural frequencies of pipes with different diameters and the same crack size are simulated and the rates of change of the natural frequencies (Shorten for FCRs) are compared. It is found that the smaller the diameter is, the more sensitive the crack is to FCRs. Then, the FCRs of the first four natural frequencies of the same pipe with different cracks are compared. The FCRs increase with the propagation of cracks. However, the curves of FCRs vibrate perpendicular to the pipe and vibration amplitudes vary with position along pipes. Therefore, it can identify crack growth, but can't locate the position of the crack by natural frequencies of small pipes. The analysis results can provide necessary theoretical basis for the identification of crack propagation in small pipes.
\end{abstract}

\section{Introduction}

The operation safe is a matter of cardinal significance in nuclear power industry. There are a lot of small branch pipes in nuclear power plants. Vibration fatigue is a principle damage of the small branch pipes and it will cause pipes to crack. Accumulation of the cracks of the pipes can cause the power plant to shut down, maintenance, and even catastrophic accidents ${ }^{[1]}$. There are many reasons that can induce the vibration of the pipes in nuclear power plants, such as the vibration of rotating equipment, hydraulic hammering action, fluid pulsation, cavitation corrosion, gas liquid two-phase flow, wind, earthquake, artificial error, et al. It is difficult to distinguish which reasons are the key factors and what are the interaction relationships among various factors, so it is a big challenge to solve vibration completely basing on theory analysis. In the interest of solve this problem, structural health monitoring and nondestructive testing are effective means to remedy the shortcoming of the theory analysis. At present, on-line fatigue monitoring system is mainly aimed at the pressured equipment. And non-destructive testing generally neglects small branches because the curvatures are too big to find suitable sensors ${ }^{[2]}$. Therefore, study on crack identification of small branch pipes is very important.

The structural damage detection method based on vibration is popular, because vibration parameters are easy to measure on the site of plants ${ }^{[3]}$. Natural frequency is a key parameter of the vibration characteristics. Therefore, many damage identification methods based on the natural frequency have been studied. Cawley and Adams have identified the damage level by the frequency changes of the flat plate before and after the generation of damage ${ }^{[4]}$. The natural frequency variation of a simple supported beam with a crack at different damage locations and different damage levels has been studied by Shang ${ }^{[5]}$. The sensitive parameters to the natural frequency of the beam have been tested, and Xuan et al have found that the geometric parameters affected the natural frequency of the cracked beam ${ }^{[6]}$. The influences of length, diameter and confinement of submarine pipeline on the natural frequency have been analyzed by $\mathrm{Yu}$ et $\mathrm{al}^{[7]}$. However, the variation laws of natural frequency with the crack propagating and geometrical parameters of cracked small pipes haven't reported yet. 
Therefore, the analysis of the changing rule of natural frequency of small cracked pipes has important theoretical significance to the damage evaluation in power plants.

\section{Finite element modeling of a cracked pipe}

A small pipe with crack is shown in Fig. 1. The pipe is simply-supported by two ends. Geometric features of cracks have been defined by location and geometrical structure. $L_{\mathrm{C}}$ is crack location, $a$ crack depth, $2 \theta$ circumferential crack length, $R$ pipe radius, $t$ wall thickness, and $L$ the length of the pipe. Pipe specimens are made from 316L stainless steel, elastic modulus $E$ is $195 \mathrm{GPa}$ and Poisson's ratio 0.3 .

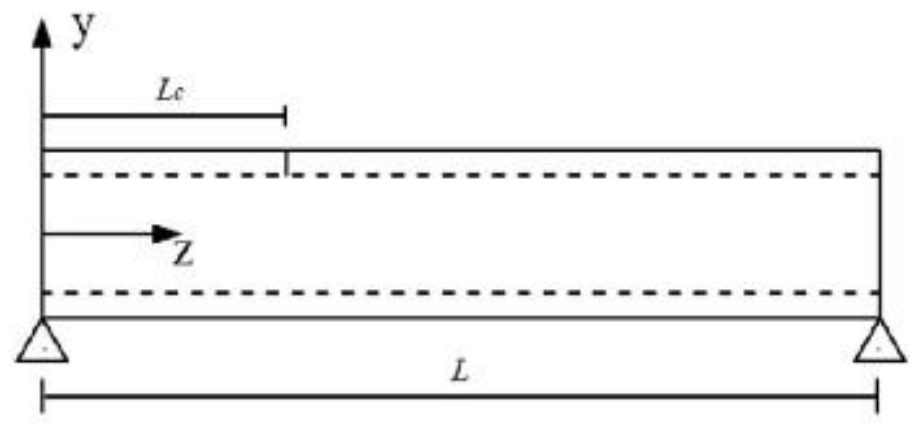

(a) Main view

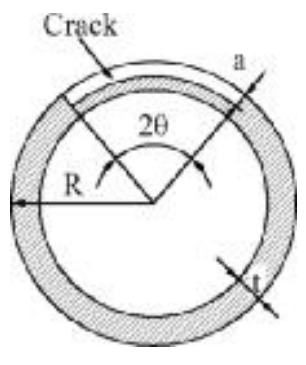

(b) left view

Fig. 1 Sketch map of crack small pipe

A cracked pipe is modeled by the finite element software ABAQUS using the C3D20R hexahedron unit. The model of the cracked pipe is established by crack zone and non-crack zone, respectively. The circumferential crack is combined by $1 / 4$ circular crack block s04_q70x2 and penetrating crack block s04_t35x 1 using crack-block ZENCRACK software. The modeling process is shown in Fig. 2.

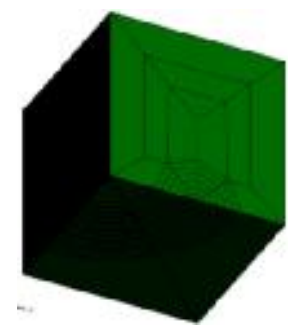

(a) $1 / 4$ circular crack block

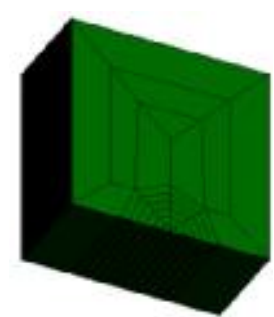

(b) penetrating crack block

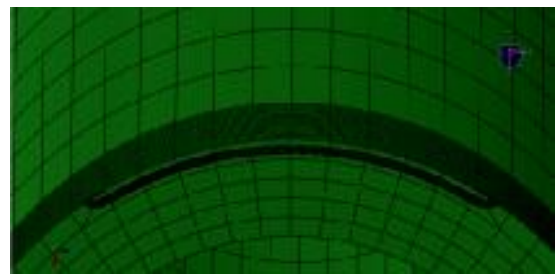

(c) The circumferential crack

Fig. 2 Cracked pipe modeling process

\section{Natural frequency analysis of cracked pipes with different diameters}

Pipe's natural frequency is quite complex. In the present study, the first four order natural frequencies are considered. In order to compare the rate of change of the natural frequency of the cracked pipes, the frequency rate of change is defined:

$$
F C R_{i}=\frac{f_{u i}-f_{d i}}{f_{u i}} \times 100 \%
$$


In Eq. $1, f_{d i}$ is the $i$ th natural frequency of the cracked pipe, and $f_{u i}$ is the $i$ th natural frequency of the pipe without crack.

In order to explore the influence of diameters on natural frequencies, five pipes with different diameters have been analyzed in this section. In the process of analyzing, all parameters have been nondimensionalized in order to simplify analysis and reduce the interference of other factors. Relative crack location $L c / L$ is selected as 0.4 , and relative crack depth $a / t$ is 0.6 . The circumferential length of the crack on the outer surface of the pipe is $40 \mathrm{~mm}$. The FCRs of the cracked pipes with different diameter are calculated, and the results are shown in Table 1.

Table 1. Variation of FCRs with different diameters

\begin{tabular}{c|ccccc}
\hline Diameter & & & & & \\
\cline { 1 - 4 } Modal $_{\mathrm{F}}[\mathrm{mm}]$ & 48 & 60 & 76 & 89 & 140 \\
\hline $1^{\text {st }}$ order & & & & & \\
$2^{\text {nd }}$ order & $0.38 \%$ & $0.30 \%$ & $0.23 \%$ & $0.19 \%$ & $0.10 \%$ \\
$3^{\text {rd }}$ order & $0.15 \%$ & $0.11 \%$ & $0.10 \%$ & $0.08 \%$ & $0.05 \%$ \\
$4^{\text {th }}$ order & $0.60 \%$ & $0.45 \%$ & $0.32 \%$ & $0.25 \%$ & $0.11 \%$ \\
\hline
\end{tabular}

According to table 1, it is easy to know that the smaller the diameter is, the larger the FCR is. That is to say, the smaller the pipe is, the more sensitive of crack pipe is to the natural frequency. Therefore, the natural frequency monitoring can be an alternative and practical method to keep the safety of small pipes, because the vibration monitoring is easy to perform.

\section{Natural frequency analysis of cracked pipes with different crack}

In order to analyze the variation rules of FCRs when cracks propagating, circumferential length of crack $\theta / \pi$ equaling to $1 / 4$ is selected. The rates of frequency change with different crack depth $a / t$ have been simulated. The curves of FCR $i$ versus crack location $L_{c} / L$ is plotted in Fig. 3.

According to Fig. 3, it can find that FCRs increase with the increase of the crack depths for the same location of the cracks. However, the curves of FCRs are nonlinear. For a giving crack depth, the curves of FCRs vibrate perpendicular to the pipe and vibration amplitudes vary with position along pipes. The number of oscillation peaks equal to the orders of the corresponding modals. That is to say, the curves of FCRs of the first modal vibrate one times and those of the second modal vibrate twice, et al. Therefore, when the vibration sensors are installed on a small pipe, the variation of the FCRs can identify the developing of cracks, but it is difficult to evaluate the exact location of the crack on the pipe.

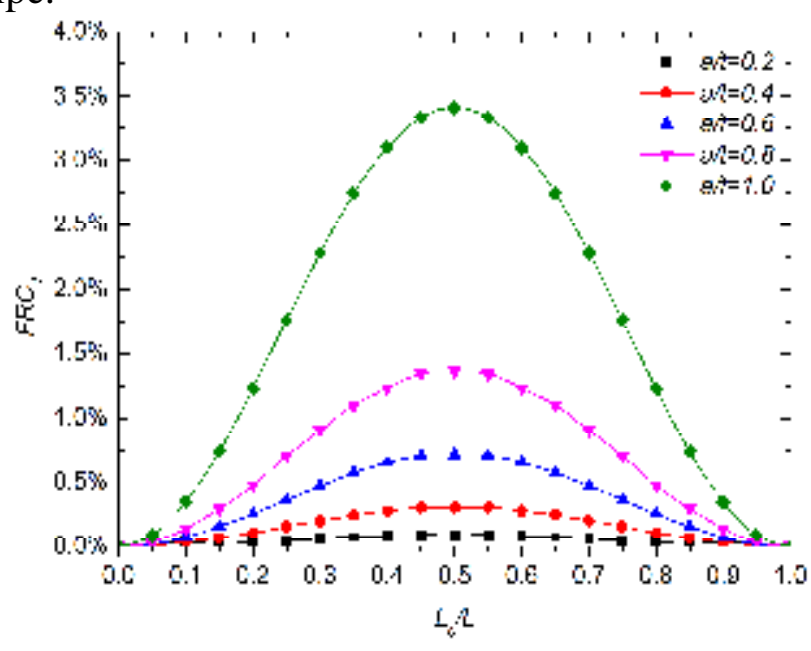

(a) the FCRs of the first natural frequency

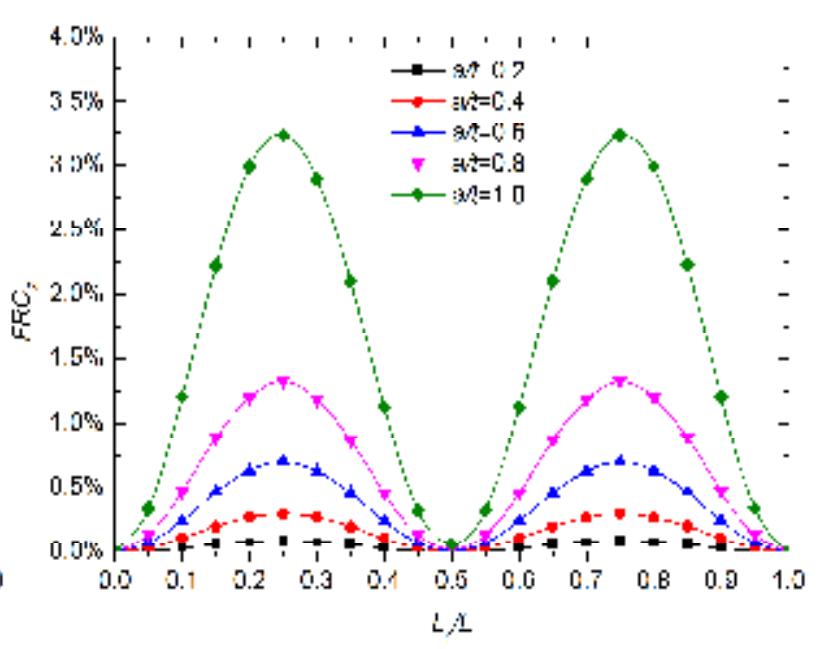

(b) the FCRs of the second natural frequency 


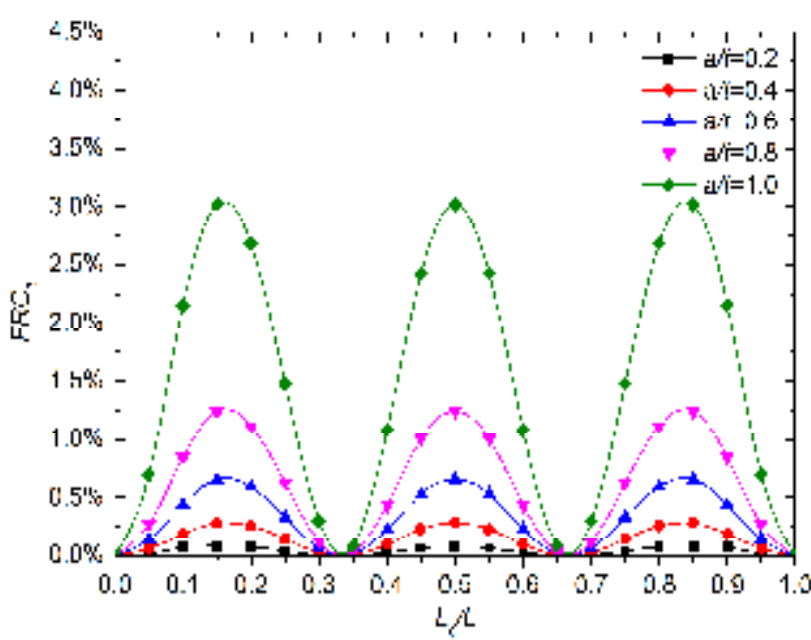

(c) the FCRs of the third natural frequency

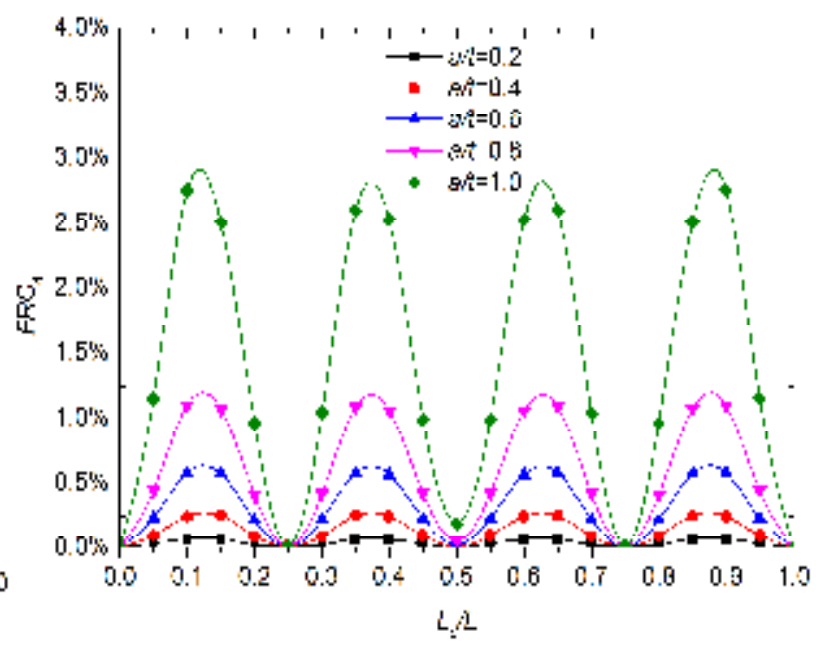

(d) the FCRs of the forth natural frequency

Fig. 3 The rates of change of natural frequency versus crack location

\section{Conclusions}

Vibration fatigue is a principle damage of the small branch pipes and it will cause pipes to crack. In order to discover an easy, cheaper and reliable method to identify the crack growth of the small pipes, the effect of the diameter, crack depth and crack position on the natural frequency of the small cracked pipes are studied by the finite element software ABAQUS. The modes of cracked pipes with different diameters and different crack sizes are setup and analyzed by finite element software. Research results show that the smaller the pipe is the more sensitive of the cracked pipe is to natural frequencies. Therefore, the natural frequency monitoring can be an alternative and practical method to keep the safety of small pipes. When cracks propagate, the rates of change of the natural frequencies increase. However, the curves of the FCRs vibrate perpendicular to the pipe and vibration amplitudes vary with position along pipes. Therefore, it can identify crack growth, but can't locate the position of the crack by natural frequencies of small pipes.

\section{Acknowledgement}

This work was financially supported by Shanghai Pujiang Program (No. 15PJD010) and the111 Project.

\section{References}

[1] H. Shiguang, S. Xiaoyao: Noise and Vibration Control. Vol. 35 (2015), p. 208 210.

[2] J. Jiuhong, Z. Chao, Z. Zhongqiang, X. Fuzhen and Z. Shaoping: Procedia Engineering. EI. Vol. 130 (2015), p. 1592 1597.

[3] G. Fangqing, J. Jianming and G. Shuying: Journal of Southwest Jiaotong University. SCI. Vol. 33 (1998), p. 108 113.

[4] P. Cawley, R.D. Adams: Journal of Strain Analysis for Engineering Design. SCI. Vol. 14 (1979), p. 49 57.

[5] S. Xin, X. Yue and R. Gengfeng: Journal of Xi'an University of Architecture and Technology (Natural Science Edition). EI. Vol. 45 (2013), p. 640 646.

[6] X. Jianping, Y. Bo and W. Jianan: Journal of Huazhong University of Science and Technology (Natural Science Edition). EI. Vol. 40 (2012), p. 1 5.

[7] Y. Jingyu, G. Weiwei, L. Xiaoyi, Z. Runwu and L. Shulin: Machinery. Vol. 53 (2015), p. 11 15. 